\title{
Low Power Wearable Audio Player Using Human Body Communications
}

\author{
Seong-Jun Song, Seung Jin Lee, Namjun Cho, and Hoi-Jun Yoo \\ Dept. of EECS, Korea Advanced Institute of Science and Technology (KAIST) \\ tornado@eeinfo.kaist.ac.kr
}

\begin{abstract}
This paper presents a prototype wearable audio player system to playback the digital audio signal transmitted through the wearer's body without any wire. To significantly reduce the power consumption of the digital audio transmission, the system exploits a novel wideband signaling human body communication scheme. The transceiver chip designed, fabricated, and assembled on the prototype system shows 5- $\mathrm{mW}$ power consumption at $2-\mathrm{Mb} / \mathrm{s}$ data rate, better than the Bluetooth transceiver. Real-time audio playing through the human body is successfully demonstrated.
\end{abstract}

\section{Introduction}

Wearable computing is emerging as a key technology that enables users to exchange multimedia information such as high quality audio and to enjoy them anywhere and anytime in ubiquitous computing environments [1]. This paper describes a prototype for the digital audio streaming through the wearer's body by exploiting the transceiver chip of [2]. Figure 1 shows an example for audio music listening by only touching the prototype system without any wire. When the finger touches the electrode on the backside of the audio player, the audio signal goes through the skin of the body to the earset on the user's ear. It can remove earphone wire and enjoy audio music comfortably and continuously. Therefore, this work shows possibility for touch-based digital audio streaming services in ubiquitous computing environments.

\section{Related Works}

There are three approaches for wearable digital audio streaming:Bluetooth, smart textiles, human body communication (HBC). The Bluetooth transceiver [3] operates at $2.4 \mathrm{GHz}$ ISM band that is very congested and prone to interference, and also consumes a huge power. Recently, a wearable digital audio player system

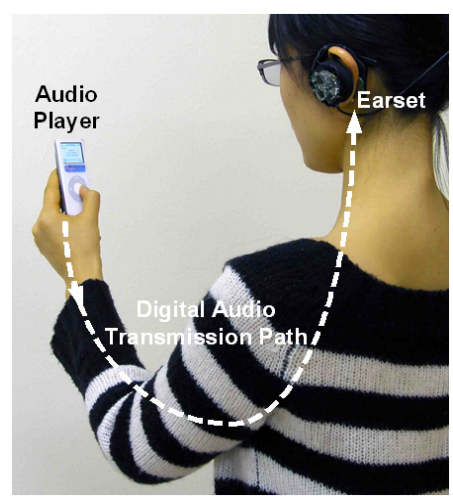

Figure 1. Photo of Real-Time Audio Playing

integrated into fashionable clothing was presented and all components are connected via the conductive textiles [4], but it needs additional manufacturing processes. Meanwhile, touch-based HBC schemes introduced in [5]-[6] face the difficulty to apply for wearable audio player applications due to limited low data rate or bulky size with high power consumption.

\section{Design Architecture}

The design architecture for our prototype system is illustrated in Figure 2, which exploits wideband signaling (WBS) for the digital audio streaming over the human body. Its architecture consists of five parts: physical medium, interface layer, signaling link layer, transceiver module, and application. The human body as the physical medium shows a wide band-pass operational spectrum and sustains the channel capacity sufficient to transfer real-time multimedia data streams as investigated in [2]. The electrical signal can be flowed by the feeble electric field induced on the skin of the body. The interface layer provides the connection with the physical medium. After the connection is established, the digital audio data transfers to the wearable I/O devices over a WBS link. Transceiver module performs the packet generation of the streaming data and supports the WBS link that 
enables a point-to-point transfer or broadcasting between each application layer.

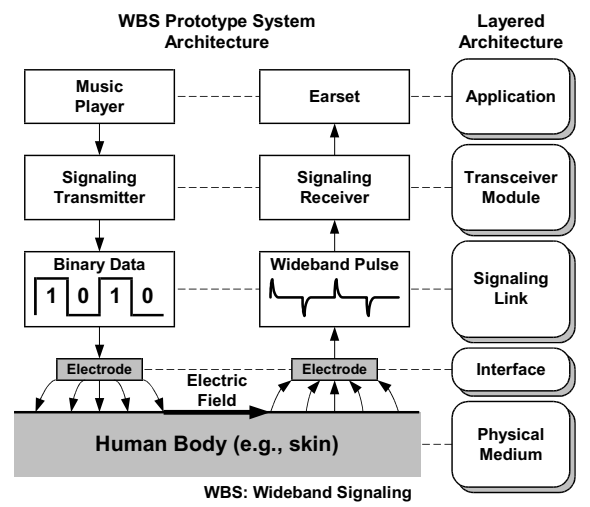

Figure 2. Design architecture for the digital audio streaming based on WBS

\section{Realization}

The prototype system is realized by using the WBS transceiver chip developed of [2]. The system hardware excluding the transceiver chip consists of off-chip components: 24-bit $\sum-\Delta$ analog-to-digital converter (ADC) and digital-to-analog converter (DAC), digital audio interface transmitter and receiver as shown in Figure 3 (a). The S/PDIF standard is chosen for the digital audio interface. The analog audio signals for two stereo channels are sampled by the 24bit sigma-delta ADC. The 24-bit sampled audio data is converted to a 32-bit packet consisting of a 4-bit preamble, the 24-bit data, and a 4-bit channel status and error detection bits. Subsequently, the data is transmitted with biphase-mark encoding at twice of the bit rate, which allows clock recovery from the data at the receiver. The effective data rate is $2.048-\mathrm{Mb} / \mathrm{s}$ at the sampling rate of $16-\mathrm{kHz}$ over the channel. The audio sound is played back by the 24-bit $\Sigma-\Delta$ DAC. No audio decoder is required at the earset. Thus, it leads to the reduction of the cost and the power consumption. The transceiver chip controls the WBS link for the transmission of digital audio data. The 0.25- $\mu \mathrm{m}$ CMOS WBS transceiver chip including a receiver AFE consumes only $5-\mathrm{mW}$ from a $1-\mathrm{V}$ supply. Compared with a Bluetooth transceiver [3], it exhibits lower power consumption at high data rate. Figure 3 (b) shows the photo of the prototype system consisting of a WBS audio transmitter connected to a MP3 player and a WBS audio receiver assembled into an earset. The transmitter attaches to the back of the audio player. The $\mathrm{Rx}$ electrode is mounted on the hanger of the earset. Measured waveforms are shown in Figure 3 (c).

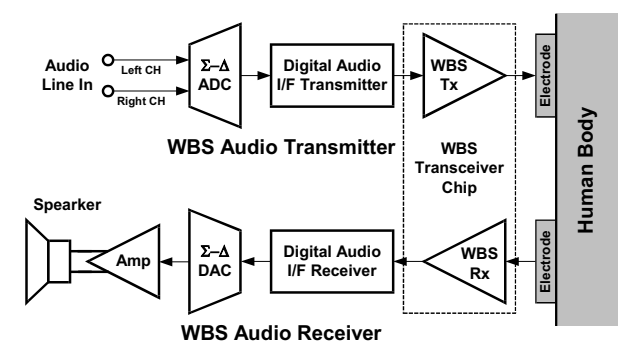

(a)

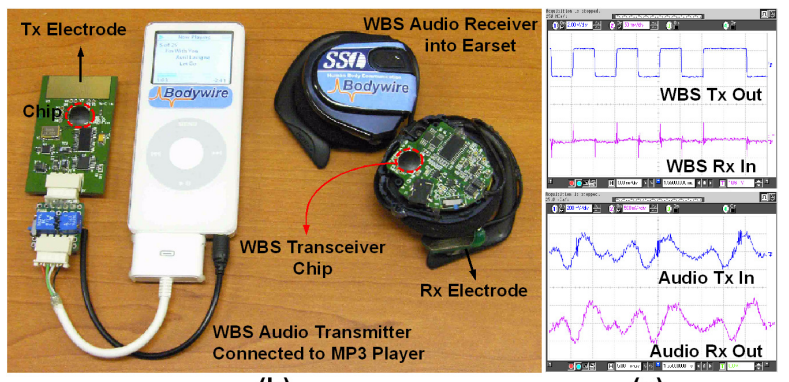

(b)

(c)

Figure 3. (a) Block diagram, (b) prototype, and (c) waveforms of the WBS audio player system

\section{Conclusion}

A wearable real-time audio player system is implemented and demonstrated to show the feasibility of the transmission of the multimedia data through the human body. The system supports the WBS link controlled by the developed $0.25-\mu \mathrm{m}$ CMOS WBS transceiver chip. It achieves low cost, and low power dissipation along with high data rate operation, compared with fabric or short-range RF solutions.

\section{References}

[1] M. Billinghurst et al., "Wearable Devices: New Ways to Manage Information," IEEE Computer, vol. 32, pp. 57-64, Jan. 1999.

[2] S.-J. Song, et al., "A 2Mb/s Wideband Pulse Transceiver with Direct-Coupled Interface for Human Body Communications," in IEEE ISSCC Dig. Tech. Papers, Feb. 2006, pp. 558-559.

[3] C. Cojocaru "Low Power Bluetooth for Headset Applications," in IDEAS'05, July 2005, pp. 223-226.

[4] S. Jung, et al., "A Digital Music Player Tailored for Smart Textiles: First Results," Avantex Symposium, 2002.

[5] N. Matsushita, et al., "Wearable Key: Device for Personalizing nearby Environment," in Proc. Int. Symp. Wearable Computer, Oct. 2000, pp. 119-126.

[6] M. Shinagawa, et al., "A Near-Field-Sensing Transceiver for Intrabody Communication Based on the Electrooptic Effect," IEEE Trans. Instrum. Meas., vol. 53, pp. 15331538, Dec. 2004. 